\title{
FOXO1-dependent DNA damage repair is regulated by JNK in lung cancer cells
}

\author{
YINGHUA JU, TAOJUN XU, HONGKAI ZHANG and AIMING YU \\ Department of Biochemistry and Molecular Biology, College of Basic Medical Sciences, \\ China Medical University, Shenyang 110001, P.R. China
}

Received December 2, 2013; Accepted December 23, 2013

DOI: $10.3892 /$ ijo.2014.2269

\begin{abstract}
DNA damage or mutation in cells contributes to tumorigenesis. The transcription factor FOXO1 modulates the expression of genes involved in DNA damage repair, cell cycle arrest and apoptosis. The transcriptional activity of FOXO1 is fundamentally regulated by post-translational modification and subcellular localization. H1299 lung cancer cells were treated with the alkylating agent MNNG, and the cell viability and DNA damage were separately determined by MTT and comet assay. Using immunofluorescence and western blotting, we observed the subcellular localization of FOXO1 and measured the relevant protein expression levels, respectively. To examine cell cycle arrest and apoptosis, flow cytometry analysis was preformed. The interaction between FOXO1 and JNK was analyzed through immunoprecipitation. Our results showed that cell viability was reduced at $24 \mathrm{~h}$ after MNNG treatment, and appeared to recover to some degree at $48 \mathrm{~h}$. The increased expression and nuclear export of FOXO1 emerged at $4 \mathrm{~h}$ after the treatment. Nuclear FOXO1 played a pivotal role in cell cycle arrest, apoptosis and DNA damage repair by upregulating $\mathrm{p} 27^{\mathrm{Kipl}}$, Bim and GADD45 gene expression, respectively. AKT-dependent S256 phosphorylation of FOXO1 and the S473 phosphorylation of AKT were both enhanced following DNA damage. Moreover, our studies revealed that FOXO1 directly interacted with JNK, and the inhibition of the JNK activity led to decreased expression of FOXO1 target genes. These findings suggest for the first time that FOXO1 is a promising candidate substrate for JNK, and the FOXO1dependent DNA damage repair may be regulated positively by the JNK pathway in H1299 lung cancer cells.
\end{abstract}

\section{Introduction}

DNA damage is constantly caused by diverse sources including endogenous metabolite and exogenous assaults (1).

Correspondence to: Professor Aiming Yu, Department of Biochemistry and Molecular Biology, College of Basic Medical Sciences, China Medical University, Shenyang 110001, P.R. China E-mail: yinghua_423@hotmail.com

Key words: FOXO1, DNA damage, MNNG, lung cancer, JNK
Of the mutagens, alkylating agents are generally unavoidable owing to their abundant presence in the environment and within living cells. By reacting with DNA bases, alkylating agents generate covalent adducts and subsequently trigger DNA strand breaks (2).

The maintenance of genome integrity and fidelity is essential for cell growth, proliferation and cell survival. While failure to repair DNA lesions may result in impairment of cellular function and in occurrence of gene mutagenesis (3). When the injure is triggered by alkylating agents, DNA damage response (DDR), as a major way to eliminate lethal and tumorigenic mutations, is initiated (4), giving rise to transcriptional activation, cell cycle arrest, DNA repair or apoptosis in cells (5). Induction of DNA damage is one of the primary mechanisms underlying the anticancer chemotherapeutic drugs, for instance cisplatin and camptothecin used in the clinic. Therefore, DNA damage response was considered as double-edged sword, on one side it affects the sensitivity to anticancer chemotherapy for cell, and on the other, perturbation of DNA repair function leads to accumulation of DNA damage, thereby resulting in genomic instability (6).

Forkhead transcription factors of the O class (FOXOs) are characterized by a conserved forkhead box DNA-binding domain, the mammalian orthologs of Caenorhabditis elegans DAF-16. FOXO subfamily is composed of four members FOXO1, FOXO3a, FOXO4, and FOXO6 (7). FOXO factors regulate a variety of physiological and pathological processes and are considered to be potential targets of tumor therapy (8). FOXO3a and FOXO4 have been shown to function as a master switch initiating G1/G2 phase arrest and DNA damage repair through upregulating $\mathrm{p} 27^{\mathrm{Kip} 1}$ and growth arrest and DNA damage inducible protein 45 (GADD45) expression (9-11). As the first identified member of the FOXO subfamily, FOXO1 has recently been demonstrated to modulate gene expression, such as $\mathrm{p} 27^{\mathrm{Kip} 1}$ and $\mathrm{p} 21^{\mathrm{cip} 1}$ involved in cell cycle arrest, GADD45 in DNA damage repair, Bim and FasL in apoptosis (12). Therefore, FOXO1 is considered to determine cell fate by a different mechanism after stress-induced damage accroding to cell types and contexts. However, what role is played in DDR by FOXO1 is not fully understood and needs to be elucidated.

The transcriptional activity of FOXO1 is tightly regulated by multiple posttranslational modifications such as phosphorylation, acetylation and ubiquitination (13). Growth 
factor stimulates activation of the phosphatidyl inositol 3-kinase (PI3K)-AKT pathway, leading to phosphorylation of nuclear FOXO1 at specific sites (Thr24, Ser256 and Ser319). Phosphorylated FOXO1 then binds to 14-3-3 chaperone proteins and becomes sequestered in the cytoplasm, which is unable to influence its target gene expression (14). In addtion, AKT-mediated phosphorylation of FOXO1 on S256 facilitates interaction with the E3 ubiquitin ligase Skp2, leading to its subsequent ubiquitination and proteasomal degradation (15). To the contrary, the activation of c-Jun N-terminal kinase (JNK) promotes transcriptional activity of FOXO in response to external stimuli. JNK-dependent T447 and T451 phosphorylation enhances the nuclear translocation thereby increasing transcriptional activity of FOXO4 (16). However, it is still unclear whether FOXO1 is a downstream effector of JNK and whether this regulation is direct as well as positive.

In the present study, we investigated the protective roles of FOXO1 in DNA damage caused by the alkylating agent in lung cancer cells. N-methyl-N'-nitro-N-nitrosoguanidine (MNNG) is a biochemical tool used experimentally as a carcinogen and mutagen. Since both FOXO and p53 transcription factors share the same target genes, including $\mathrm{p} 21^{\text {cip1 }}$ and GADD45 (17), the p53-deficient cell line H1299 was selected to avoid p53 interference in the study. Our data show that FOXO1 affects the output of DDR by facilitating nuclear import and upregulation of target gene expression in H1299 cells. FOXO1dependent cell cycle arrest and apoptosis were observed during MNNG-inflicted DNA damage. In addition, our results show that FOXO1 formed a complex with JNK kinase during DNA damage in H1299 cells. Furthermore, when using JNK inhibitor its activity was suppressed, nuclear exclusion of FOXO1 was examined, and the decrease in expression levels of FOXO1 target genes was measured. The results confirmed that FOXO1 participates in the cellular responses to genotoxic stimuli, and suggests that JNK make a significant contribution to alteration in FOXO1 transcriptional activity during DDR in H1299 cells.

\section{Materials and methods}

Cell culture and cytotoxicity assay. Human lung adenocarcinoma cancer cells H1299 and human bronchial epithelial (HBE) cells were maintained in high-glucose DMEM medium with $10 \%$ fetal bovine serum (Gibco, CA, USA), penicillin $(100 \mathrm{U} / \mathrm{ml})$ and streptomycin $(100 \mu \mathrm{g} / \mathrm{ml})$. In certain experiments, JNK inhibitor SP600125 (Sigma, St. Louis, MO, USA), was added. All cells were incubated at $37^{\circ} \mathrm{C}$ in $5 \% \mathrm{CO}_{2}$.

For cell viability assay, H1299 cells were seeded in 96-well plates at $5 \times 10^{3}$ cells/well. Cells were allowed to adhere for $24 \mathrm{~h}$, and subconfluent cells were treated with MNNG (0.5-10 $\mu \mathrm{M})$ (Sigma) for $30 \mathrm{~min}$. Then, the cells were washed with phosphate-buffered saline (PBS) three times to remove the alkylating agents. Cell growth inhibitory studies were carried out following incubating for 24 or $48 \mathrm{~h}$, and cells were tested by MTT (3-(4,5-dimethylthiazol-2-yl)-2,5- biphenyl tetrazolium bromide) assay.

Alkaline comet assay. The alkaline comet assay was conducted according to the Tice et al analyzing method (18). After exposure to 1-10 $\mu \mathrm{M}$ MNNG for $30 \mathrm{~min}$, H1299 cells were collected and resuspended in cold PBS. The cells in the $0.5 \%$ low melting point agarose were placed on a slide precoated with a layer of $1 \%$ regular agarose. These two layers were solidified at $4^{\circ} \mathrm{C}$, and immersed in a cold lysing solution (100 mM Na $\mathrm{mDTA}_{2}, 2.5 \mathrm{M} \mathrm{NaCl}, 10 \mathrm{mM} 1 \%$ Triton $\mathrm{X}-100,10 \%$ DMSO, Tris- $\mathrm{HCl}, \mathrm{pH} 10.5)$ for $1 \mathrm{~h}$ at $4^{\circ} \mathrm{C}$. Subsequently, the gel slides were dried and soaked in fresh electrophoresis solution ( $1 \mathrm{mM} \mathrm{Na}{ }_{2}$ EDTA, $300 \mathrm{mM} \mathrm{NaOH}$, $\mathrm{pH} 13.0$ ) for $20 \mathrm{~min}$. Electrophoresis was run at $300 \mathrm{~mA}$, $25 \mathrm{~V}$ for $20 \mathrm{~min}$ at $4^{\circ} \mathrm{C}$. After staining with ethidium bromide $(20 \mu \mathrm{g} / \mathrm{ml})$ for $10 \mathrm{~min}$, the slides were neutralized with $0.4 \mathrm{M}$ Tris- $\mathrm{HCl}(\mathrm{pH} 7.5)$. The slides were washed with water and observed under a fluorescent microscope (BX51; Olympus, Tokyo, Japan). The parameters representing DNA damage were obtained and recorded for 100 cells per sample by CASP software.

Immunofluorescence. Cells grown on coverslips were fixed in $4 \%$ paraformaldehyde for $15 \mathrm{~min}$, and permeabilized with $0.5 \%$ Triton $\mathrm{X}-100$ for $30 \mathrm{~min}$ at room temperature. Subsequently, the cells were blocked with 3\% BSA for $30 \mathrm{~min}$, and incubated with monoclonal anti-FOXO1 antibody (1:100, Cell Signaling Technology, Boston, MA, USA) overnight at $4^{\circ} \mathrm{C}$. These cells was incubated with tetramethylrhodamine (TRITC)-5-(and-6)-isothiocyanate)-conjugated secondary antibody for $1 \mathrm{~h}$. Finally, the slides were stained with DAPI solution for $15 \mathrm{~min}$ at $37^{\circ} \mathrm{C}$ following rinsing with PBS. Digital images were examined under a fluorescence microscope (BX61; Olympus).

FACS analysis. For cell cycle and apoptosis analysis, subconfluent cells were treated with 0.1-5 $\mu \mathrm{M}$ MNNG for $30 \mathrm{~min}$, and incubation was continued with growth medium for $24 \mathrm{~h}$. In order to analyze the cell cycle, harvested cells were fixed in $70 \%$ ethanol overnight at $4^{\circ} \mathrm{C}$, followed by rinsing with cold PBS. Resuspended in PBS solution, cells were incubated with RNase I $(10 \mathrm{~g} / \mathrm{l})$ for $30 \mathrm{~min}$ at $37^{\circ} \mathrm{C}$ and stained with $50 \mathrm{mg} / \mathrm{ml}$ propidium iodide (PI) for $30 \mathrm{~min}$ in the dark. The DNA content was evaluated by flow cytometry (Becton-Dickinson, NJ, USA). For analysis of apoptosis, cells were harvested and washed with PBS, then stained with Annexin V-FITC for $10 \mathrm{~min}$ and $50 \mathrm{mg} / \mathrm{ml}$ propidium iodide for $5 \mathrm{~min}$ successively. Apoptotic cells were assessed by outer membrane phosphatidylserine translocation as well as nuclear fluorescence.

Immunoblot and immunoprecipitation analysis. Cell extracts were prepared with RIPA lysis buffer adding $0.5 \%$ protease inhibitor cocktail and $1 \%$ phosphatase inhibitors. Protein samples were separated on 10 or $12 \%$ SDS-PAGE gel and transferred for $2.5 \mathrm{~h}$ to PVDF membranes. Blots were then probed ordinally with primary antibody according to the respective titers provided by their manufacturers, and with appropriate secondary antibody, then visualized using enhanced chemiluminescence reagent (Sigma). Membranes were blocked in 5\% non-fat dry milk and washed in TTBS. Anti-FOXO1 was purchased from Cell Signaling Technology. Anti-AKT, anti-p-AKT Ser 473, anti-p-FOXO1 Ser 256 and anti-FOXO4, anti-p-JNK, antiGADD45, anti-Skp2 and anti-normal rabbit IgG, anti-Bim, 


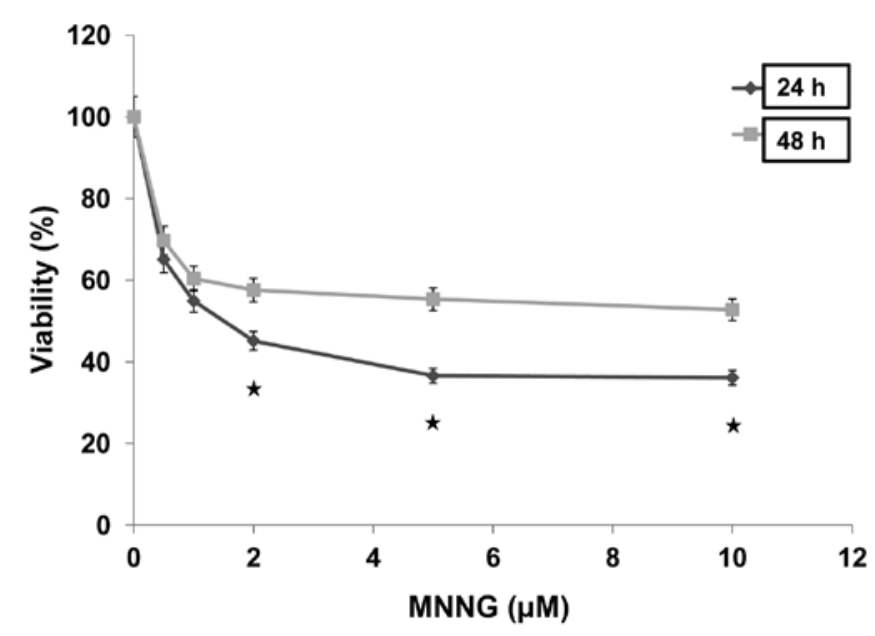

Figure 1. Inhibition of cell growth and DNA damage induced by MNNG. H1299 cells were treated with MNNG $(0,0.5,1,2,5$ and $10 \mu \mathrm{M})$ for 30 min followed by incubation with growth medium for 24 or $48 \mathrm{~h}$. Viability was assessed using MTT assay. The results are from three independent experiments. Each value represents the mean of five replicates \pm standard error of the mean. ${ }^{*} \mathrm{p} \leq 0.05$ significant difference between cells cultured for 24 and $48 \mathrm{~h}$.

anti-p27, anti- $\beta$-actin and anti-GAPDH were purchesed from Santa Cruz Biotechnology (Santa Cruz, CA, USA).

For immunoprecipitation analysis, cell lysates were precleared with Protein G Plus agarose for $30 \mathrm{~min}$ at $4^{\circ} \mathrm{C}$. Primary antibody or rabbit normal $\mathrm{IgG}$ and Protein $\mathrm{G}$ agarose beads ( $40 \mu \mathrm{l}$ of $50 \%$ bead slurry, Santa Cruz Biotechnology) were then added to $500 \mu \mathrm{l}$ cell lysates for 2 -h incubation at $4^{\circ} \mathrm{C}$. Following five washings with cold lysis buffer, immunoprecipitates were analyzed by western blotting in $10 \%$ gel.

\section{Results}

MNNG inhibits cell growth by inducing DNA damage in H1299 cells. In order to investigate the cytotoxic effect of MNNG on cell viability, H1299 cells were incubated in the presence of increasing concentrations of $\mathrm{MNNG}$ for $30 \mathrm{~min}$, then maintained in growth medium for 24 or $48 \mathrm{~h}$. MTT assay demonstrated MNNG inhibited cell growth in a dose-dependent manner in H1299 cells (Fig. 1). The percentages of viable cells cultured for 24 and $48 \mathrm{~h}$ after treatment with $0.5 \mu \mathrm{M}$ MNNG remarkably reduced to 65.1 and $69.7 \%$ compared with control, respectively, and ultimately reduced to the plateau phases (36.6 and 55.3\%) after treatment with $5 \mu \mathrm{M}$ MNNG. In addition, cells kept cultured for $24 \mathrm{~h}$ after treated with MNNG showed lower viability than those cultured for $48 \mathrm{~h}$ after similar treatment. MNNG was tested at a continuous cell exposure dose of $5 \mu \mathrm{M}$ with the experiments carried out at 0.5 to 10 , and $5 \mu \mathrm{M}$ was selected for further analysis. These data indicated that cells may have the ability to self-repair in response to $\mathrm{MNNG}$-induced damage.

MNNG is a wildly used alkylating agent considered to cause methylation of DNA. To understand the mechanism underlying MNNG-induced cytotoxicity in H1299 cells, we employed the alkaline comet assay, because this assay is a sensitive and reliable technique for evaluating the presence and level of DNA strand breaks (19). As shown by results of the comet assay, fluorescence intense increased in the comet tail, revealing the the fragments of DNA were excluded from their nuclei, while the control cells displayed only fluorescent nuclei without comet tails, after exposure to various concentrations of MNNG (0, 1, 5 and $10 \mu \mathrm{M})$ for $30 \mathrm{~min}$ (Fig. 2). A statistically significant difference was observed when these data from the exposed group was compared to that from the control group. This difference was manifested in the values of three comet parameters. The difference of average value in tail length, tail intensity and tail moment between cells of the exposed and the control group were $52.1 \pm 10.1 \mu \mathrm{m}$, $9.04 \pm 2.21 \%$ and $3.31 \pm 1.63(\mathrm{p}<0.001)$, respectively. The data of MTT and comet assay revealed that MNNG inhibits cell growth by inducing DNA strand breaks in a dose-dependent manner. Hence, we speculated that the partial recovery of cell viability in p53-null H1299 cells might be attributed to FOXO1-mediated DNA repair response. Thus, it is essential to further define the function of FOXO1 during DNA damage response.

DNA damage enhances the protein expression and nuclear import of FOXO1. The expression levels and subcellular localization of FOXO are two key factors influencing their transcriptional activity. Increase in the protein level in the vicinity of the nucleus contributes to the nuclear translocation of FOXO factors, and intranuclear FOXOs can promote their target genes transcription. Based on this, we first detected a change in its protein level after MNNG treatment, to see if FOXO1 is involved in DNA damage repair in H1299 cells. Of note is the significant increase in FOXO1 expression using western blotting was observed at $4 \mathrm{~h}$ after MNNG treatment, thus the analyses described below were carried out at this time-point. Western blotting showed that significant increase in the expression level of FOXO1 was found at various concentrations but not at $10 \mu \mathrm{M}$ (Fig. 3A). This result indicates that tolerable DNA damage can induce FOXO1 expression, while excessive cell injury suppresses FOXO1 expression.

Transcriptional factor FOXO1, when maintained in the nucleus, is involved in negative regulation of cell cycle progression. We were interested to see whether MNNG treatment give rise to nuclear translocation of FOXO1 in H1299 cells. Therefore, immunofluorescence investigation was carried out to visualize the localization of FOXO1. Cells were subjected to $0-5 \mu \mathrm{M}$ MNNG treatment for $30 \mathrm{~min}$ and subsequent culture for $4 \mathrm{~h}$ prior to staining and microscopic examination. As shown in Fig. 3B, the endogenous FOXO1 distributed almost completely in the cytoplasm in untreated H1299 cells, and nuclear staining was negligible. Compared with the control, nuclear fluorescence was distinctly enhanced, indicating that FOXO1 proteins significantly accumulated in the nuclei, in H1299 cells treated with increasing doses of MNNG. These results provide evidence that nuclear translocation of FOXO1 takes place as a response to MNNG. Results of western blotting and immunofluorescence were in agreement, DNA damage promotes the transcriptional activity of FOXO1 in H1299 cells.

FOXO1 is involved in DNA damage response by enhancing target gene expression. To further investigate the role of FOXO1 in DNA damage response, we also examined its target 

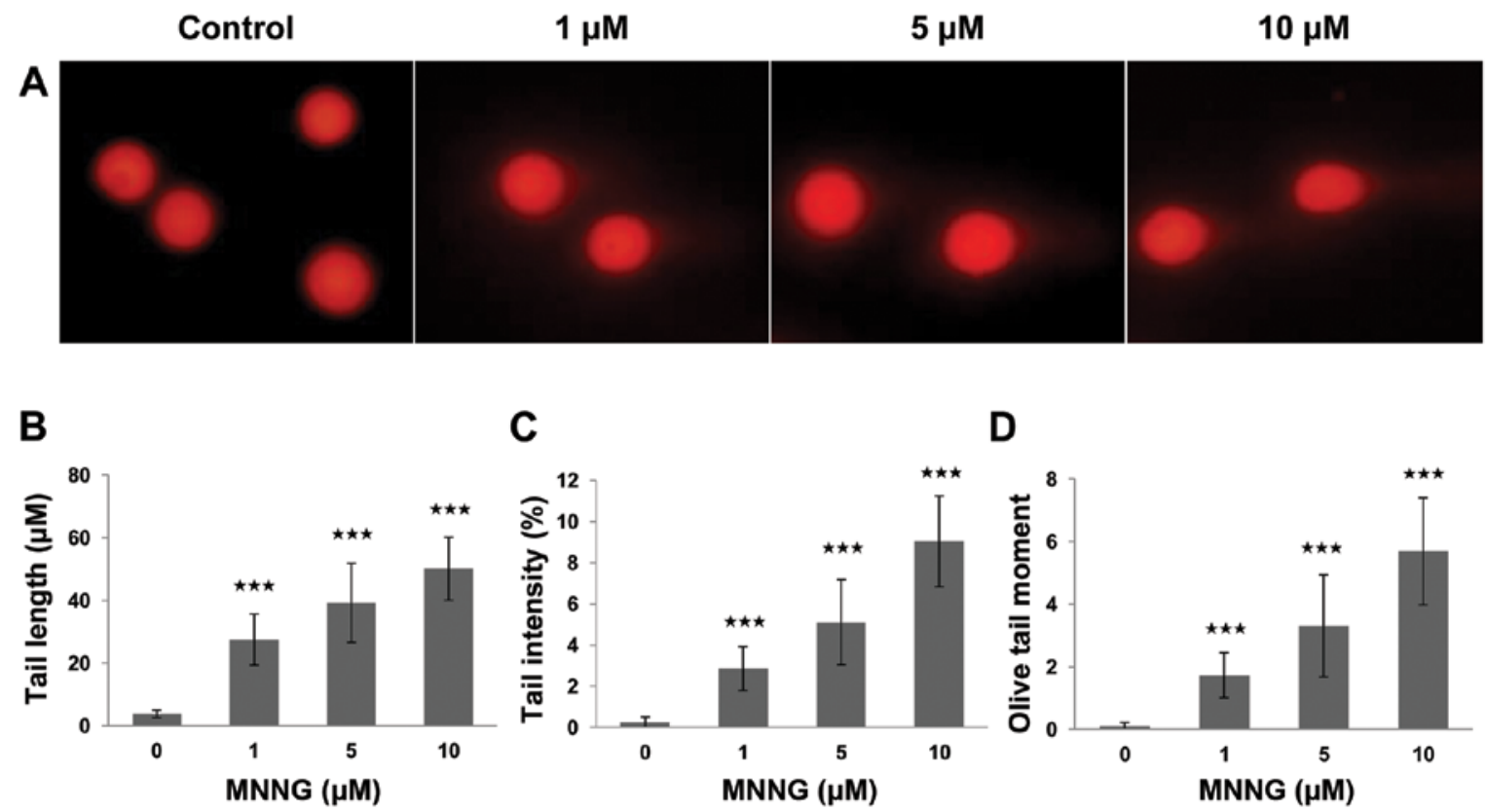

Figure 2. The extent of DNA damage of H1299 cells induced by MNNG in various concentrations. H1299 cells were treated with various concentrations of MNNG for $30 \mathrm{~min}$. (A) The levels of DNA damage were examined by comet assay using fluorescence microscopy. Data are representative of three independent experiments. (B-D) The mean of the parameters were analyzed from 100 independent cells per sample by Casp software. The tail length $=$ length of the comet tail, the tail intensity = percent of DNA in the comet tail. The Olive tail moment = tail intensity $x$ (tail DNA mean-head DNA mean). Data denoted ${ }^{*} \mathrm{p} \leq 0.05,{ }^{* *} \mathrm{p} \leq 0.01$ and ${ }^{* * *} \mathrm{p} \leq 0.001$ are significant compared to untreated control.

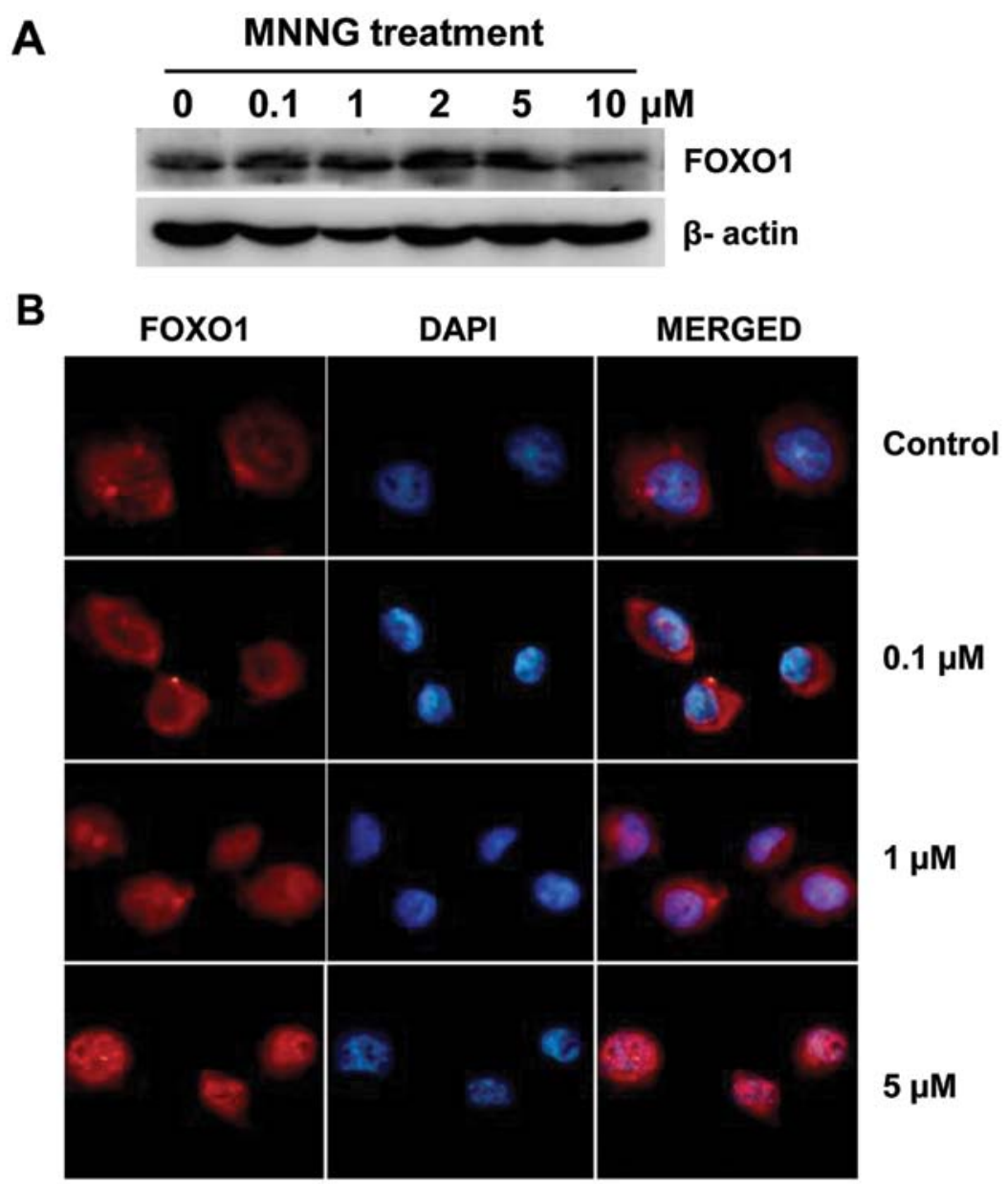

Figure 3. The protein expression and nuclear import of FOXO1 were both enhanced by MNNG induced DNA damage. H1299 cells were treated with various concentrations of MNNG for 30 min followed by incubation with growth medium for $4 \mathrm{~h}$. (A) The expression levels of FOXO1 and $\beta$-actin were monitored by western blotting using the appropriate antibodies. Data are representative of at least three independent experiments. (B) Treated cells were fixed and immunostained with anti-FOXO1 antibody. The sublocalization of FOXO1 was analyzed by fluorescence microscopy. 
A

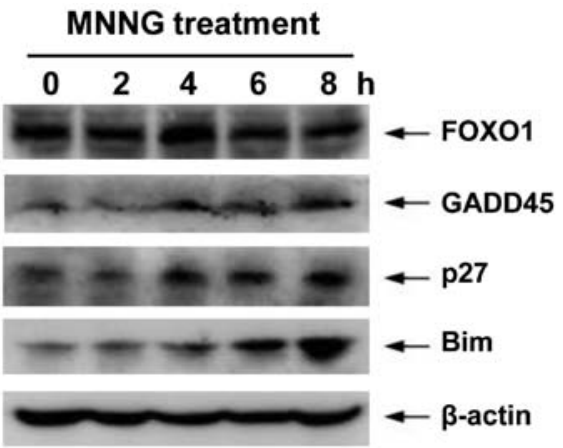

B

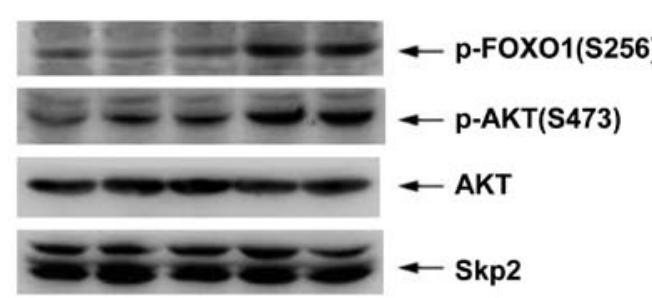

Figure 4. Protein expression of FOXO1 target genes and PI3K/AKT pathway during DNA damage. H1299 cells were incubated with $5 \mu \mathrm{M}$ MNNG for $30 \mathrm{~min}$. Thereafter, cells were incubated in growth medium for $0,2,4,6$ or $8 \mathrm{~h}$ before lysis. FOXO1, p27 ${ }^{\text {Kipl }}$, GADD45, Bim, p-FOXO1 (S256), p-AKT (S473), AKT, Skp2 and $\beta$-actin protein levels were determined by western blotting using the appropriate antibodies. Data are representative of at least three independent experiments. genes, such as p27 ${ }^{\mathrm{Kip} 1}$, GADD45 and Bim by western blotting after MNNG treatment. Commonly, confronted with a variety of stresses involving DNA damage, cells can rapidly take protective responses. After treatment with $5 \mu \mathrm{M}$ MNNG for $30 \mathrm{~min}, \mathrm{H} 1299$ cells were incubated in growth medium for different time-points $(0,2,4,6$ and 8 h). As shown in Fig. 4A, the transient increase in FOXO1 expression was observed at $4 \mathrm{~h}$ after MNNG treatment, but the content of FOXO1 declined to the original level $6 \mathrm{~h}$ later. Simultaneously, the expression of p27 ${ }^{\mathrm{Kip} 1}$, GADD45 and Bim displayed a continuous upregulation from 4 to $8 \mathrm{~h}$ after MNNG treatment. Thus, the induced p2 $7^{\text {Kip1 }}$, GADD45 and Bim proteins serve as regulators of the cell cycle, DNA repair and apoptosis to execute their functions. These data demonstrate further that FOXO1 is involved in DNA damage response by modulating target gene expression in H1299 cells.

It is known that AKT-dependent phosphorylation of FOXO1 on S256 may lead to nuclear exclusion of FOXO1, and subsequently Skp2 protein mediates FOXO1 degradation through ubiquitin-proteasome system. Therefore, to study the effect on the transcriptional activity of FOXO1 via the PI3K-AKT pathway, we further determined the expression or activity of associated proteins using western blotting during DNA damage response in H1299 cells. These results showed that both the phosphorylation levels of AKT on S473 (AKT
A

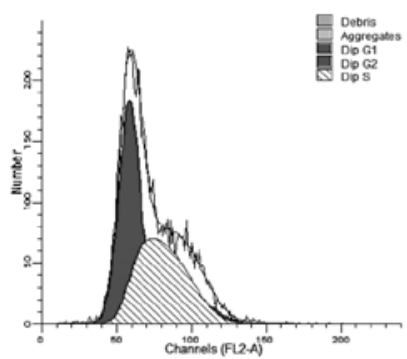

G

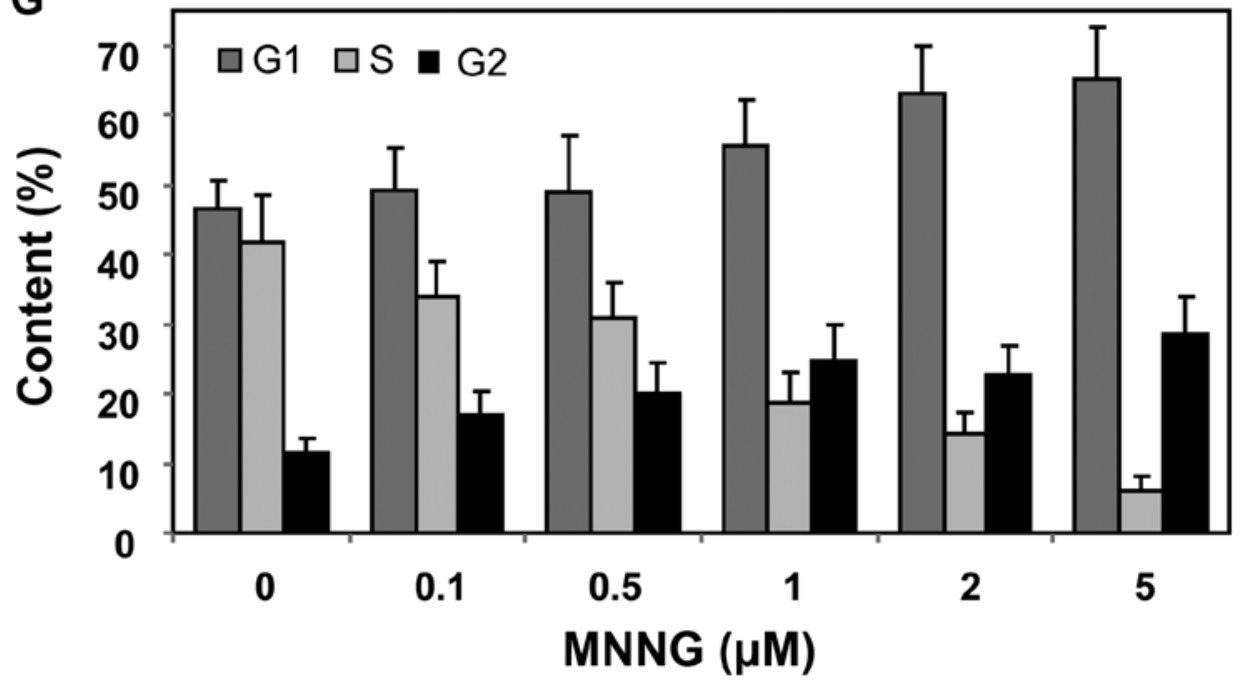

B

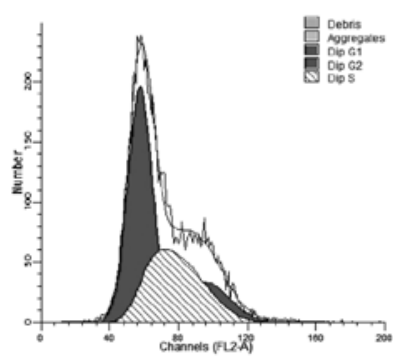

C

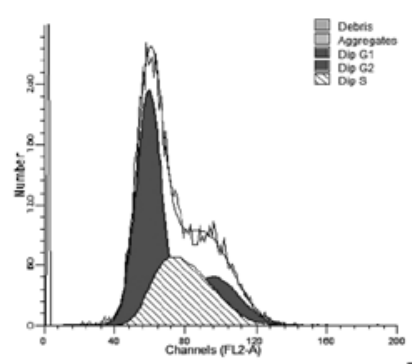

E
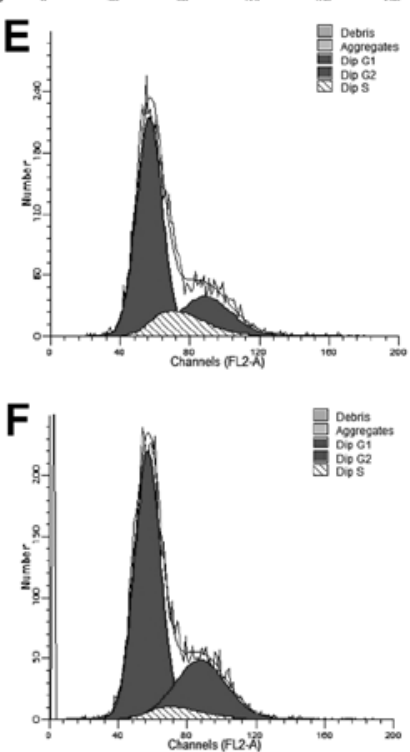

Figure 5. The cell cycle arrests at G1 and G2 phase in response to DNA damage. (A-F) H1299 cells were treated with various concentrations of MNNG $(0,0.1$, $0.5,1,2$ and $5 \mu \mathrm{M}$ ) for $30 \mathrm{~min}$. After subsequently culture for $24 \mathrm{~h}$, cells were stained with PI and analyzed for DNA content by flow cytometry. Results are representative of three separate experiments. (G) Percentage of cells in each phase (G1, S or G2) are presented. Data represent the mean \pm SD of three independent experiments. 

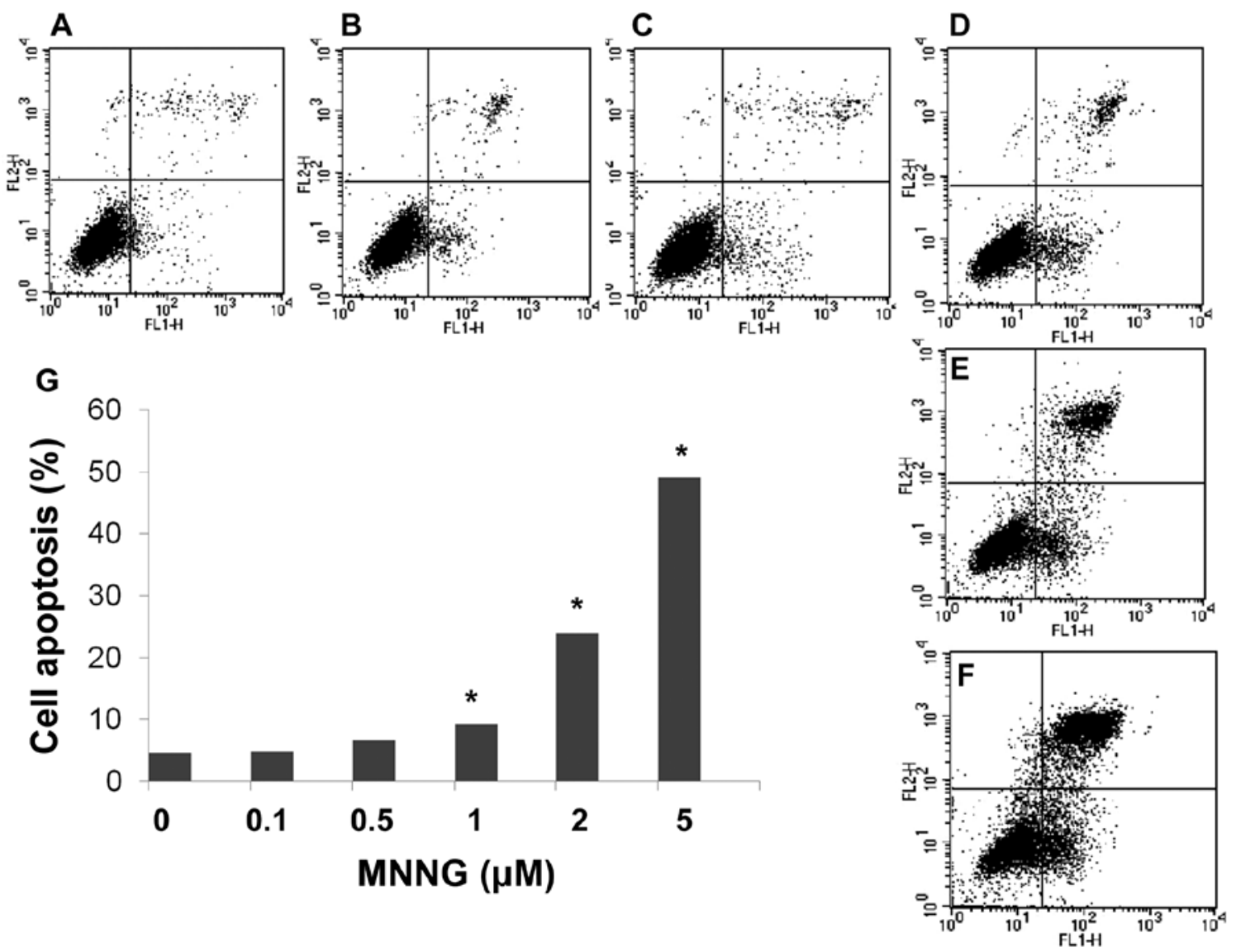

Figure 6. Apoptosis is induced during FOXO1 mediated DNA damage response. (A-F) H1299 cells were incubated with MNNG in various concentrations $(0,0.1,0.5,1,2$ and $5 \mu \mathrm{M})$ for 30 min followed by culture in growth medium for $24 \mathrm{~h}$, prior to detection of apoptosis using cyclin A/propidium iodide staining by flow cytometry. Two independent experiments produced similar results. (G) The percent of apoptotic cells was determined as Annexin V-positive/ propidium iodide-negative stained cells, bars represent mean $\pm \mathrm{SD}$. ${ }^{*} \mathrm{p} \leq 0.05$.

active form) and FOXO1 on S256 mediated by AKT significantly increased at 6-8 h after MNNG treatment, coinciding with the reduction of FOXO1 content. However, the MNNG exposure did not cause changes in total proteins of AKT and Skp2 (Fig. 4B).

FOXO1-induced cell cycle arrest responds to DNA damage. When DNA damage is induced, the cell cycle progression will be blocked to provide time for repairing DNA damage. Since FOXO1 increased the expression levels of $\mathrm{p} 27^{\mathrm{Kip} 1}$ and GADD45, cell cycle should be arrested, thereby repairing DNA damage. In order to further examine the DNA repair effect connected with FOXO1, we analyzed cell cycle after DNA damage in $\mathrm{H} 1299$ cells. The results showed that the cells arrested in G1 and G2 phase were significantly increased from 45.1 and $12.4 \%$ to 65.6 and $28.5 \%$, respectively, after $5 \mu \mathrm{M}$ MNNG exposure (Fig. 5). Taken together, these data display that FOXO1 negatively regulates cell cycle progression, and facilitates DNA damage repair by modulating expression of p2 ${ }^{\mathrm{Kip} 1}$ and GADD45 in response to MNNG in H1299 cells.

FOXO1 induces apoptosis during DNA damage. Bim is a FOXO1 target gene serving as a proapoptotic protein. We found that expression level of Bim increased in a dose-dependent manner in H1299 cells. Moreover, flow cytometric analysis using Annexin V and PI double staining further confirmed apoptosis occurring after MNNG treatment. These results show that the number of apoptotic cells significantly increased dose-dependently after exposure to various doses of MNNG for 30 min (Fig. 6). Thus, results of flow cytometric analysis are consistent with detection using western blotting.

JNK improves the transcriptional activity of FOXO1 by facilitating nuclear translocation. JNK, also known as stressactivated protein kinase (SAPK), involves cellular response to a variety of external stimuli. To define whether JNK pathway was also involved in the response to MNNG-inflicted DNA damage in H1299 cells, we examined the expression level of p-JNK (T183, Y185) at various time-points. As shown in Fig. 7A, phosphorylation status of JNK was transiently enhanced at 2 and $4 \mathrm{~h}$ after MNNG treatment in H1299 cells, and high activity phosphorylation status was maintained for $4 \mathrm{~h}$ in HBE cells. Therefore, FOXO1 total protein level correspondingly increased at $4 \mathrm{~h}$ in $\mathrm{H} 1299$ cells and at 2 and $4 \mathrm{~h}$ in HBE cells (Figs. 2A and 7A). These data imply that activation of JNK may be involved in regulation of transcriptional activity of FOXO1. Thus, to determine the role of JNK in MNNG response, we carried out immunoprecipitation analysis. The activated JNK associated with FOXO1 (Fig. 7B), suggesting FOXO1 also served as a substrate for JNK. Moreover, inhibition of JNK activity by SP600125 (5 and $10 \mu \mathrm{M})$ reduced the nuclear translocation of FOXO1 (Fig. 7D). In addition, 
A

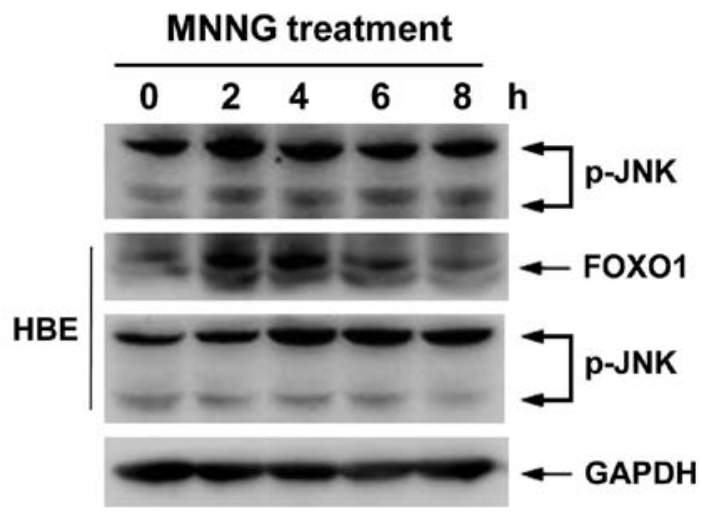

B IP: MNNG treatment

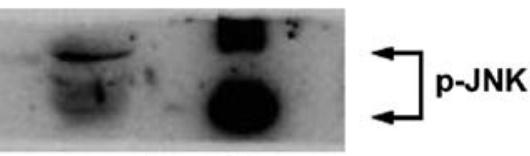

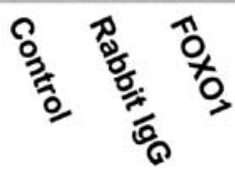

D

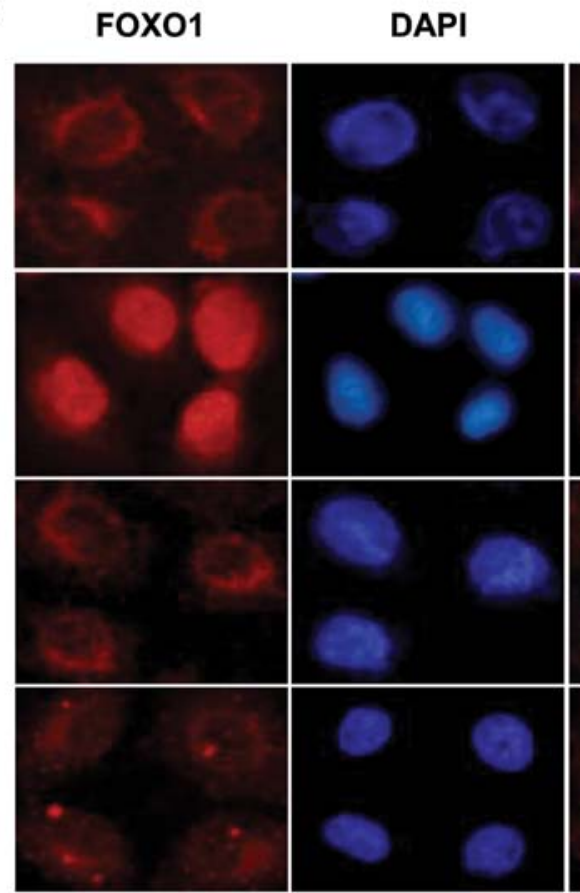

C

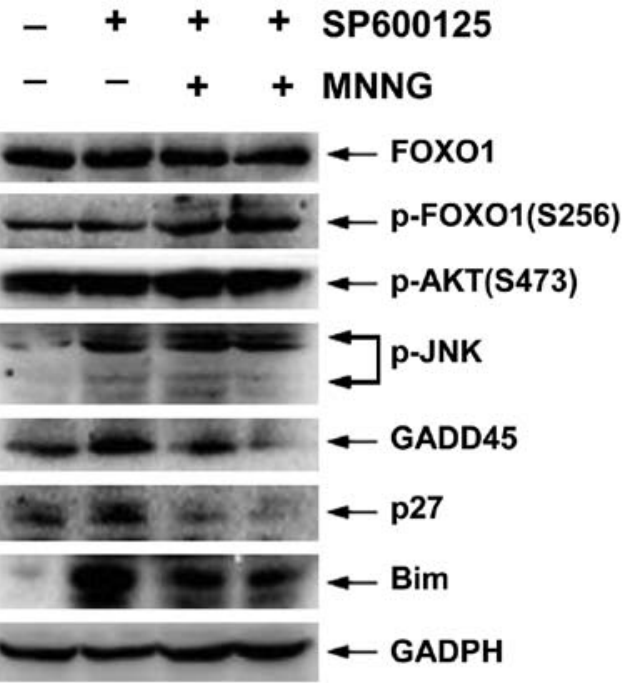

MERGED

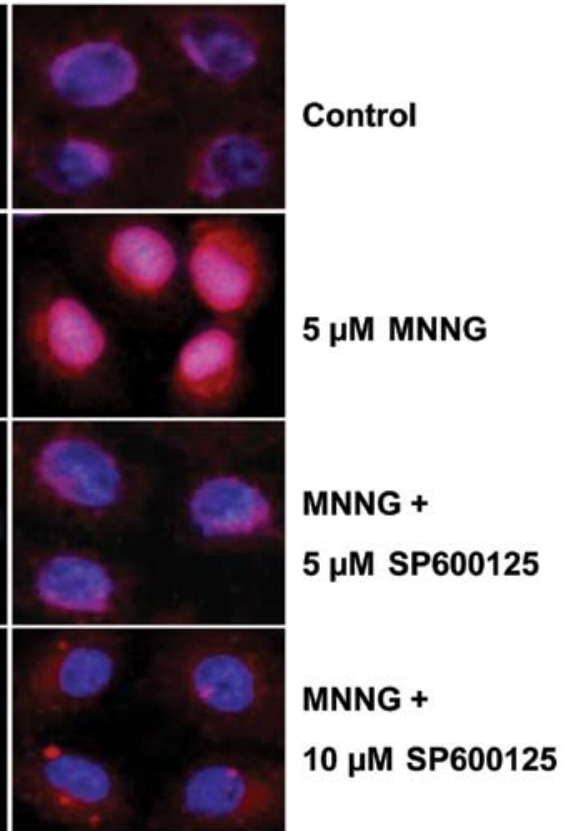

Figure 7. The c-Jun N-terminal kinase (JNK) activation may be required for the FOXO1-dependent repair response of DNA damage. (A) H1299 and HBE cells were incubated with $5 \mu \mathrm{M}$ MNNG for $30 \mathrm{~min}$ followed by culture with growth medium for $4 \mathrm{~h}$ before lysis. Target proteins were determined by western blotting. (B) H1299 cells were treated in the same condition, binding of endogenous FOXO1 to p-JNK was assayed by immunoprecipitation of FOXO1 followed by western blotting using an antibody directed against p-JNK. (C) H1299 cells were pretreated with $5,10 \mu \mathrm{M}$ or without JNK pathway inhibitor SP600125 for 2 h, then incubated with or without $5 \mu \mathrm{M}$ MNNG for $30 \mathrm{~min}$ followed by incubating with growth medium for $4 \mathrm{~h}$, target proteins were determined by appropriate antibodies. (D) H1299 cells were treated as described in (C), then fixed and immunostained with anti-FOXO1 antibody. The sublocalization of FOXO1 was visualized by fluorescence microscopy.

we found that SP600125 could suppress the expression levels of FOXO1 target genes, such as GADD45, p27 and Bim. However, it showed no significant change in the levels of either FOXO1 total protein or p-AKT (S473) (Fig. 7C). These results demonstrated that JNK can function as an important regulator in MNNG response, promoting nuclear translocation and target gene expression of FOXO1 by physical interaction with FOXO1 in H1299 cells. 


\section{Discussion}

DNA damage can be caused by multiple stimuli from the environment including chemical mutagens. Following genotoxic stress, an inherent DNA damage response (DDR) is initiated to eliminate DNA lesions. The DDR serves as a network of molecular signalling events that modulate DNA repair, cell cycle arrest and apoptosis (5). In this study, we adopted MNNG as an alkylating agent that induces chemical DNA damage, as the molecular mechanism of FOXO1-mediated DNA repair response in p53-deficient cells is understood. We have found that treatment of H1299 cells by short-term MNNG results in a dose-dependent inhibition of proliferation. Interestingly, viability of H1299 cells displayed a recovery at $48 \mathrm{~h}$ after MNNG exposure in comparison with $24 \mathrm{~h}$, suggesting that MNNG-inflicted DNA damage might be defused gradually. It is known that alkylating agent-induced genotoxicity is mainly due to triggering of DNA single-strand or double-strand breaks. They react with the ring nitrogens $(\mathrm{N})$ and extracyclic oxygen $(\mathrm{O})$ atoms of DNA bases to generate a variety of covalent adducts (20). Therefore, we further analyzed the levels of MNNG-caused DNA damage by assessing the content of DNA strand breaks in H1299 cells using the comet assay. As cellular DNA damage becomes serious, more DNA fragments migrate into the comet tail region, and are quantified by the intensity of fluorescence according to comet parameters including tail length, tail intensity and Olive tail moment, representing the levels of DNA damage (21). Our results exhibited significant increase in these three parameters following MNNG treatment, revealing that MNNG gave rise to DNA strand breaks in a dose-dependent manner. This confirmed that a decline in cell viability attributed to DNA damage occurred after MNNG treatment.

In response to DNA strand breaks, cells undergo corresponding DNA repair process such as homologous recombination repair (HRR) or the non-homologous endjoining (NHEJ) $(22,23)$. It is well known that ATM and ATR play critical roles in DNA damage response by phosphorylating CHK1, CHK2 and p53 $(24,25)$. FOXO proteins as transcription factors improve expression of a number of genes that are involved in cell cycle, DNA repair and cell death. The recovery of cell viability implies that MNNG-induced DNA damage might be partly repaired in H1299 cells. Therefore, we assumed that FOXO1-dependent DNA repair may be subsequently induced after MNNG treatment. In this study, H1299 lung adenocarcinoma cells deficient in p53 gene were utilized, thereby preventing p53 interference, and is thus appropriate for investigating the role of FOXO1 in DNA damage repair. We show for the first time that FOXO1 protein mediates DNA repair response by facilitating its protein expression and nuclear translocation, and then promoting expression of target genes in $\mathrm{H} 1299$ cells. Indeed, p27 ${ }^{\mathrm{Kipl}}$ and GADD45 expression can block the cell cycle and subsequently initiate DNA repair, respectively. $\mathrm{p} 27^{\mathrm{Kip} 1}$, a well-known cyclin-dependent kinase inhibitor (CKI), can induce G1 phase arrest by inhibiting CDK-mediated phosphorylation of Rb protein (26). Growth arrest and DNA damage inducible protein 45 (GADD45) is involved in DNA repair and G2 phase arrest (27). Our results demonstrate that MNNG treatment of H1299 cells induced cell cycle arrest in either G1 or G2 phase, this coincides with the inducing of $\mathrm{p} 27^{\mathrm{Kipl}}$ and GADD45. These results are consistent with the literature on FOXO3a which describe G1 and G2 arrest induced by DNA damage in cancer cells $(9,10)$. In addition, we observed an increase in Bim expression after MNNG treatment. Bim is a proapoptotic member of the Bcl-2 family, and ectopic expression of Bim is sufficient for triggering apoptosis in a variety of cell types (28). Apoptosis is induced after MNNG treatment, suggesting that FOXO1 is involved in cell apoptosis in response to MNNG-mediated DNA damage in H1299 cells. Our results are in agreement with the report of Nakamura and Sakamoto, showing that FOXO1 plays an essential role in reactive oxygen species-induced apoptosis by enhancing expression of Bim in HeLa cells (29). Cell cycle arrest and apoptosis response appear to be two critical mechanisms for eliminating DNA lesions, the former provides time for repairing DNA damage, and the latter prevents cells from passing on errant genetic codes that can lead to disease. Based on the above, it is suggested that FOXO1 can determine cell fate from survival to death by modulating expression of different target genes based on the cell context.

The activity of FOXO1 is mainly in control of signal pathway-dependent posttranslational modification, particularly phosphorylation. Although the role of FOXO1 in multiple cellular events such as proliferation, cell cycle arrest, apoptosis and oxidative stress has been well studied (30), the regulatory mechanism of FOXO1 in response to DNA damage is relatively unclear. Therefore, we further investigated the relevant signal pathways involved in FOXO1dependent DNA damage repair.

Since FOXO1 plays an important role in cell cycle arrest, DNA repair and apoptosis during MNNG response in H1299 cells, it is necessary to understand the regulatory mechanism of FOXO1. In addition to expression level, the transcriptional activity of FOXO1 is mainly regulated by posttranslational modification, especially signal pathway-dependent phosphorylation. It has been reported that PI3K-AKT signaling pathway is activated and contributes to abolish FOXO-dependent cell cycle arrest in hematopoietic cells during DNA damage (12). Besides, insulin treatment decreases endogenous FOXO1 proteins via proteasomal degradation in HepG2 cells, and the degradation is mediated by the PI3K-AKT pathway $(31,32)$, indicating that PI3K-AKT pathway may be a negative regulator of FOXO1 in response to DNA damage. Therefore, we examined the levels of AKT-phosphorylated FOXO1 on S256 and relevant proteins of PI3K/AKT pathway in H1299 cells. As shown in the results, phosphorylation levels of both FOXO1 and AKT were improved during MNNG-generated DNA damage, but the total FOXO1 expression was at its former level at the same time. These observations suggest that PI3K/AKT pathway might restrain DNA repair by causing the degradation of FOXO1.

In contrast, c-Jun-N-terminal kinase (JNK) pathway appears to play a key role in response to environmental stresses including DNA damage (33). It is established that JNK could directly phosphorylate DAF-16 and FOXO4, thereby regulating their transcriptional activity, however, association between JNK and FOXO1 is as yet unidentified $(18,34)$. Here, we observed an increase in level of activated JNK (pT183, pY185) by western blotting after MNNG treatment in both H1299 and HBE cells (Fig. 7A). Furthermore, the result by 
immunoprecipitation analysis showed that JNK could directly interact with FOXO1 (Fig. 7B), suggesting FOXO1 may be a phosphorylated substrate for JNK. In Drosophila, JNK has been demonstrated to promote the nuclear translocation and target gene expression of FoxO1 (35). Using inhibitor of JNK, we observed that inhibition of JNK suppressed the nuclear translocation of FOXO1 preventing its target gene expression. These data suggest that FOXO1 serving as a substrate, may be involved in JNK pathway-mediated DNA repair response. Thus, FOXO1-dependent DNA damage repair is regulated by JNK.

In conclusion, these findings suggest for the first time that FOXO1 is a promising candidate substrate for JNK, and FOXO1-dependent of DNA damage repair may be regulated by JNK pathway positively in lung cancer H1299 cells.

\section{References}

1. Hoeijmakers JH: DNA damage, aging, and cancer. N Engl J Med 361: 1475-1485, 2009.

2. Sedgwick B, Bates PA, Paik J, Jacobs SC and Lindahl T: Repair of alkylated DNA: recent advances. DNA Repair 6: 429-442, 2007.

3. Maher RL, Branagan AM and Morrical SW: Coordination of DNA replication and recombination activities in the maintenance of genome stability. J Cell Biochem 112: 2672-2682, 2011.

4. Fu D, Calvo JA and Samson LD: Balancing repair and tolerance of DNA damage caused by alkylating agents. Nat Rev Cancer 12: 104-120, 2012.

5. Zhou BB and Elledge SJ: The DNA damage response: putting checkpoints in perspective. Nature 408: 433-439, 2012.

6. Lam M, Carmichael AR and Griffiths HR: An aqueous extract of Fagonia cretica induces DNA damage, cell cycle arrest and apoptosis in breast cancer cells via FOXO3a and p53 expression. PLoS One 7: e40152, 2012.

7. Greer EL and Brunet A: FOXO transcription factors at the interface between longevity and tumor suppression. Oncogene 24: 7410-7425, 2005.

8. Maiese K, Chong ZZ, Shang YC and Hou J: Clever cancer strategies with FoxO transcription factors. Cell Cycle 7: 3829-3839, 2008.

9. Tran H, Brunet A, Grenier JM, Datta SR, Fornace AJ Jr, DiStefano PS, Chiang LW and Greenberg ME: DNA repair pathway stimulated by the Forkhead transcription factor FOXO3a through the Gadd45 protein. Science 296: 530-534, 2002.

10. Lei H and Quelle FW: FOXO transcription factors enforce cell cycle checkpoints and promote survival of hematopoietic cells after DNA damage. Mol Cancer Res 7: 1294-1303, 2009.

11. de Keizer PL, Packer LM, Szypowska AA, Riedl-Polderman PE, van den Broek NJ, de Bruin A, Dansen TB, Marais R, Brenkman AB and Burgering BM: Activation of forkhead box $\mathrm{O}$ transcription factors by oncogenic BRAF promotes p21cip1-dependent senescence. Cancer Res 70: 8526-8536, 2010 .

12. Huang $\mathrm{H}$ and Tindall DJ: Dynamic FoxO transcription factors. J Cell Sci 120: 2479-2487, 2007.

13. Yang JY and Hung MC: A new fork for clinical application: targeting forkhead transcription factors in cancer. Clin Cancer Res 15: 752-757, 2009.

14. Vogt PK, Jiang H and Aoki M: Triple layer control: phosphorylation, acetylation and ubiquitination of FOXO proteins. Cell Cycle 4: 908-913, 2005.

15. Rena G, Prescott AR, Guo S, Cohen P and Unterman TG: Roles of the forkhead in rhabdomyosarcoma (FKHR) phosphorylation sites in regulating 14-3-3 binding, transactivation and nuclear targeting. Biochem J 354: 605-612, 2001.
16. Essers MA, Weijzen S, Vries-Smits AM, Saarloos I, de Ruiter ND, Bos JL and Burgering BM: FOXO transcription factor activation by oxidative stress mediated by the small GTPase Ral and JNK. EMBO J 23: 4802-4812, 2004.

17. Fu $\mathrm{Z}$ and Tindall DJ: FOXOs, cancer and regulation of apoptosis. Oncogene 27: 2312-2319, 2008.

18. Tice RR, Agurell E, Anderson D, Burlinson B, Hartmann A, Kobayashi H, Miyamae Y, Rojas E, Ryu JC and Sasaki YF: Single cell gel/comet assay: guidelines for in vitro and in vivo genetic toxicology testing. Environ Mol Mutagen 35: 206-221, 2000.

19. Dhawan A, Bajpayee M and Parmar D: Comet assay: a reliable tool for the assessment of DNA damage in different models. Cell Biol Toxicol 25: 5-32, 2009.

20. Drabløs F, Feyzi E, Aas PA, Vaagbø CB, Kavli B, Bratlie MS, Peña-Diaz J, Otterlei M, Slupphaug G and Krokan HE: Alkylation damage in DNA and RNA - repair mechanisms and medical significance. DNA Repair 3: 1389-1407, 2004.

21. Cok I, Ulutaş OK, Okuşluk O, Durmaz E and Demir N: Evaluation of DNA damage in common carp (Cyprinus carpio L.) by comet assay for determination of possible pollution in Lake Mogan (Ankara). Sci World J 11: 1455-1461, 2011.

22. Schroering AG and Williams KJ: Rapid induction of chromatinassociated DNA mismatch repair proteins after MNNG treatment. DNA Repair 7: 951-969, 2008.

23. Jasin M: Homologous repair of DNA damage and tumorigenesis: the BRCA connection. Oncogene 21: 8981-8993, 2002.

24. Abraham RT: Cell cycle checkpoint signaling through the ATM and ATR kinases. Genes Dev 15: 2177-2196, 2001.

25. Matsuoka S, Ballif BA, Smogorzewska A, McDonald ER III, Hurov KE, Luo J, Bakalarski CE, Zhao Z, Solimini N, Lerenthal Y, Shiloh Y, Gygi SP and Elledge SJ: ATM and ATR substrate analysis reveals extensive protein networks responsive to DNA damage. Science 316: 1160-1166, 2007.

26. Hirano M, Hirano K, Nishimura J and Kanaide H: Transcriptional up-regulation of p27 (Kip1) during contact-induced growth arrest in vascular endothelial cells. Exp Cell Res 271: 356-367, 2001.

27. Hildesheim J and Fornace AJ Jr: Gadd45a: an elusive yet attractive candidate gene in pancreatic cancer. Clin Cancer Res 8: 2475-2479, 2002.

28. Stahl M, Dijkers PF, Kops GJ, Lens SM, Coffer PJ, Burgering BM and Medema RH: The forkhead transcription factor FoxO regulates transcription of $\mathrm{p} 27^{\mathrm{Kipl}}$ and $\mathrm{Bim}$ in response to IL-2. J Immunol 168: 5024-5031, 2002.

29. Nakamura $T$ and Sakamoto K: Forkhead transcription factor FOXO subfamily is essential for reactive oxygen species-induced apoptosis. Mol Cell Endocrinol 281: 47-55, 2008.

30. Maiese K, Chong ZZ, Hou J and Shang YC: The 'O' class: crafting clinical care with FoxO transcription factors. Adv Exp Med Biol 665: 242-260, 2009.

31. Matsuzaki H, Daitoku H, Hatta M, Tanaka K and Fukamizu A: Insulin-induced phosphorylation of FKHR (Foxol) targets to proteasomal degradation. Proc Natl Acad Sci USA 100: 11285-11290, 2003.

32. Davis RJ: Signal transduction by the JNK group of MAP kinases. Cell 103: 239-252, 2000.

33. Lüpertz R, Chovolou Y, Unfried K, Kampkötter A, Wätjen W and Kahl R: The forkhead transcription factor FOXO4 sensitizes cancer cells to doxorubicin-mediated cytotoxicity. Carcinogenesis 29: 2045-2052, 2008.

34. Wang MC, Bohmann D and Jasper H: JNK extends life span and limits growth by antagonizing cellular and organism-wide responses to insulin signaling. Cell 121: 115-125, 2005.

35. Hong YK, Lee S, Park SH, Lee JH, Han SY, Kim ST, Kim YK, Jeon S, Koo BS and Cho KS: Inhibition of JNK/dFOXO pathway and caspases rescues neurological impairments in Drosophila Alzheimer's disease model. Biochem Biophys Res Commun 419: 49-53, 2012. 